The stronger result of 3.3 shows that $l(r, \theta ; \phi \bar{\phi})$ is of class PL; whence

$$
\begin{aligned}
& {[l(r, \theta ; \phi \bar{\phi})]^{2} \Delta \log l(r, \theta ; \phi \bar{\phi})} \\
& \quad=\sum_{\theta, h, j, k=1}^{\infty} \frac{a_{g} \bar{a}_{h} a_{j} \bar{a}_{k}(g-h)^{2}(j-k)^{2}}{(g+h+1)(j+k+1)(g+k+1)(h+j+1)} \\
& \quad \cdot r^{g+h+j+k} e^{i(g-h+j-k)} \geqq 0 .
\end{aligned}
$$

On the other hand, it can be shown directly, by an extension of the above identities, that (17) is positive definite.

The Rice Institute

\title{
SOME ITERATED INTEGRALS IN THE FRACTIONAL CALCULUS
}

\section{S. MACPHAIL}

1. Introduction. A considerable amount of attention has been devoted to integrals of fractional order, both in regard to their applications and to the conditions for their existence.* We shall denote the fractional integral of order $\alpha$ by

$$
{ }_{T} I_{t}^{\alpha} f(t)=\frac{1}{\Gamma(\alpha)} \int_{T}^{t}(t-v)^{\alpha-1} f(v) d v, \quad \alpha>0, t>T,
$$

and it is the purpose of this paper to give some formulas which may be of use in manipulating these integrals. We shall prove that under certain conditions the following relations hold:

$$
\begin{aligned}
\text { (2) } \int_{T}^{\infty} \frac{{ }_{T} I_{t}^{\alpha} f(t)}{t^{k+\alpha}} d t & =\frac{\Gamma(k)}{\Gamma(k+\alpha)} \int_{T}^{\infty} \frac{f(t)}{t^{k}} d t, & \alpha>0, \\
\text { (3) } \int_{T}^{\infty} e^{-k t}{ }_{T} I_{t}^{\alpha} f(t) d t & =k^{-\alpha} \int_{T}^{\infty} e^{-k t} f(t) d t, & \alpha>0, \\
\text { (4) } \int_{T}^{\infty} \cos k t_{T} I_{t}{ }^{\alpha} f(t) d t & =k^{-\alpha} \int_{T}^{\infty} \cos (k t+\pi \alpha / 2) f(t) d t, & 0<\alpha<1,
\end{aligned}
$$

and (4) holds when cosine is replaced by sine. As an application we

* A bibliography is given by H. T. Davis, Application of fractional operators to functional equations, American Journal of Mathematics, vol. 49 (1927), pp. 123-142. See also J. D. Tamarkin, On integrable solutions of Abel's integral equation, Annals of Mathematics, (2), vol. 31 (1930), pp. 219-229. 
shall evaluate by a new method certain well known integrals involving Bessel functions. It will be noticed that when $\alpha=1$ the formulas (2) and (3) agree with the results of integrating by parts in the ordinary way; they may therefore be regarded as extensions to fractional orders of integration of the familiar formula for integration by parts. The vanishing of the integrated term leads to some general theorems which are stated as corollaries to Theorem 1 below.

2. The proofs of the above formulas. The first formula may be stated in a theorem as follows:

Theorem 1. Assume $T \geqq 0, \alpha>0, k>0$. Then

$$
\int_{T}^{\infty} \frac{{ }_{T} I_{t}^{\alpha} f(t)}{t^{k+\alpha}} d t=\frac{\Gamma(k)}{\Gamma(k+\alpha)} \int_{T}^{\infty} \frac{f(t)}{t^{k}} d t
$$

provided either that the right-hand side exists in the Lebesgue sense, or that $f(t)$ is continuous and the right-hand side convergent, not necessarily absolutely.

Proof. We have

$$
\begin{aligned}
\Gamma(\alpha) \int_{T}^{\mu} \frac{{ }_{T} I_{t^{\alpha}} f(t)}{t^{k+\alpha}} d t & =\int_{T}^{\mu} \frac{d t}{t^{k+\alpha}} \int_{T}^{t}(t-v)^{\alpha-1} f(v) d v \\
& =\int_{T}^{\mu} f(v) d v \int_{v}^{\mu} \frac{(t-v)^{\alpha-1}}{t^{k+\alpha}} d t * \\
& =\int_{T}^{\mu} f(v) d v \int_{v}^{\mu} t^{-k-1}(1-v / t)^{\alpha-1} d t \\
& =\int_{T}^{\mu} \frac{f(v)}{v^{k}} d v \int_{v / \mu}^{1} u^{k-1}(1-u)^{\alpha-1} d u
\end{aligned}
$$

on setting $v / t=u$. If the right-hand side of (5) is absolutely convergent, we may let $\mu$ become infinite, justifying the process by inverting the order of integration, taking the positive and negative parts of $f(v)$ separately, and using the theorem of monotonic convergence; $\dagger$ our theorem then follows on evaluation of the $u$ integral. Otherwise a more elaborate process is necessary. We integrate by parts, setting

$$
\int_{T}^{v} \frac{f(v)}{v^{k}} d v=F(v)
$$

* The inversion is easily justified. See, for example, E. C. Titchmarsh, The Theory of Functions, p. 398, example 25.

$\dagger$ Titchmarsh, loc. cit., p. 346. 
This gives

$$
\left[F(v) \int_{v / \mu}^{1} u^{k-1}(1-u)^{\alpha-1} d u\right]_{T}^{\mu}+\frac{1}{\mu} \int_{T}^{\mu} F(v)(v / \mu)^{k-1}(1-v / \mu)^{\alpha-1} d v .
$$

The integrated term vanishes at both limits; and on the assumption that $F(v)$ approaches a limit, say $V$, as $v \rightarrow \infty$, we shall now show that the last integral approaches

$$
V \frac{\Gamma(k) \Gamma(\alpha)}{\Gamma(k+\alpha)}
$$

as $\mu \rightarrow \infty$. Write $F(v)=V+h(v)$, where $|h(v)|<\epsilon$ if $v>v_{0}$, and in any case $|h(v)|<A$ ( $A$ denoting a constant, not necessarily the same each time it is used). We divide the integral into four parts as follows. In the first place,

$$
\begin{aligned}
\frac{1}{\mu} \int_{0}^{\mu} V(v / \mu)^{k-1}(1-v / \mu)^{\alpha-1} d v & =\frac{V}{\mu^{k+\alpha-1}} \int_{0}^{\mu} v^{k-1}(\mu-v)^{\alpha-1} d v \\
& =V \frac{\Gamma(k) \Gamma(\alpha)}{\Gamma(k+\alpha)} .
\end{aligned}
$$

Also, if $\mu>T$,

$$
\frac{1}{\mu} \int_{0}^{T} V(v / \mu)^{k-1}(1-v / \mu)^{\alpha-1} d v=\frac{V}{\mu^{k}} \int_{0}^{T} v^{k-1}(1-v / \mu)^{\alpha-1} d v<\frac{A}{\mu^{k}} .
$$

Supposing further that $\mu>v_{0}>T$, we write

$$
\frac{1}{\mu} \int_{T}^{v_{0}}|h(v)|(v / \mu)^{k-1}(1-v / \mu)^{\alpha-1} d v<\frac{A}{\mu^{k}} \int_{T}^{v_{0}} v^{k-1}(1-v / \mu)^{\alpha-1} d v<\frac{A}{\mu^{k}},
$$

and finally,

$$
\frac{1}{\mu} \int_{v_{0}}^{\mu}|h(v)|(v / \mu)^{k-1}(1-v / \mu)^{\alpha-1} d v<\epsilon \frac{\Gamma(k) \Gamma(\alpha)}{\Gamma(k+\alpha)} .
$$

The theorem now follows on combining the results of the four preceding equations.

Corollary 1. If the integral

$$
\int_{T}^{\infty} \frac{f(v)}{v^{k}} d v
$$

converges, then 


$$
\frac{1}{\mu^{k}} \int_{T}^{\mu} f(v) d v \rightarrow 0
$$

as $\mu \rightarrow \infty$.

For, if $F(v)$ denotes an indefinite integral of $f(v)$, we have, on integrating by parts in the usual way,

$$
\int_{T}^{\mu} \frac{f(v)}{v^{k}} d v=\left[\frac{F(v)}{v^{k}}\right]_{T}^{\mu}+k \int_{T}^{\mu} \frac{F(v)}{v^{k+1}} d v .
$$

Comparing this with the result of the above theorem (with $\alpha=1$ ), we see that the integrated term must vanish at $\mu=\infty$, which proves the corollary. That the converse proposition need not be true may be seen by taking $f(v)=(\log v)^{-1}, k=1$.

This corollary leads us to the following more general result:

Corollary 2. If the integral

$$
\int_{T}^{\infty} g(v) f(v) d v
$$

converges, then

$$
g(\mu) \int_{T}^{\mu} f(v) d v \rightarrow 0
$$

as $\mu \rightarrow \infty$, the function $g(v)$ being any function that tends monotonically to zero as $v \rightarrow \infty$.

Suppose first that $g(v)$ is monotonically decreasing in the strict sense, and set $g(v)=u^{-1}$. Let the inverse relation be $v=G(u)$, and suppose further that $G(u)$ is the integral of a function $G^{\prime}(u)$; then by the conditions of this corollary the integral

$$
\int_{1 / g(T)}^{\infty} u^{-1} f\{G(u)\} G^{\prime}(u) d u
$$

exists.* Hence by Corollary 1 (with $k=1$ ) we have

$$
\mu^{-1} \int_{1 / g(T)}^{\mu} f\{G(u)\} G^{\prime}(u) d u \rightarrow 0 .
$$

Changing the variable back to $v$, we have

$$
\mu^{-1} \int_{T}^{G(\mu)} f(v) d v \rightarrow 0,
$$

\footnotetext{
* Titchmarsh, loc. cit., p. 377.
} 
or, what is the same thing,

$$
g(\mu) \int_{T}^{\mu} f(v) d v \rightarrow 0
$$

as required. Next, suppose that $g(v)$ is a step function tending to zero. Evidently we may replace $g(v)$ by a steadily decreasing function (in the strict sense) $\bar{g}(v)$, such that

$$
\left|\int_{T}^{\infty} g(v) f(v) d v-\int_{T}^{\infty} \bar{g}(v) f(v) d v\right|<\epsilon
$$

for any value of $\epsilon>0$, and at the same time,

$$
\lim _{v \rightarrow \infty} \bar{g}(v) / g(v)=1
$$

The corollary will then be true for $\bar{g}(v)$ and therefore for $g(v)$. Finally, let $g(v)$ be any function tending monotonically to zero, not necessarily in the strict sense. Then we can replace $g(v)$ by a step function and this in turn by a steadily decreasing function in the strict sense. The corollary now follows as before.

Corollary 3 . $^{*}$ Let $t_{1}, t_{2}, \cdots$ be a sequence tending monotonically to zero, and let $u_{1}, u_{2}, \cdots$ be any sequence such that $\sum_{n=1}^{\infty} t_{n} u_{n}$ exists. Then

$$
t_{m} \sum_{n=1}^{m} u_{n} \rightarrow 0
$$

as $m \rightarrow \infty$.

For each sum may be represented as the integral of a step function. The result then follows from Corollary 2.

Theorem 2. Assume $\alpha>0, k>0$. Then

$$
\int_{T}^{\infty} e^{-k t} T_{t}^{\alpha} f(t) d t=k^{-\alpha} \int_{T}^{\infty} e^{-k t} f(t) d t,
$$

provided either that the right-hand side exists in the Lebesgue sense, or that $f(t)$ is continuous and the right-hand side convergent, not necessarily absolutely.

Proof. We have the following relations:

* A particular case of this corollary, with $t_{n}=n^{-k}$, is given by Pólya and Szegö, Aufgaben und Lehrsätze, vol. 1, part 1, exercise 75. 


$$
\begin{aligned}
\Gamma(\alpha) \int_{T}^{\mu} e^{-k t} I_{t}^{\alpha} f(t) d t & =\int_{T}^{\mu} e^{-k t} d t \int_{T}^{t}(t-v)^{\alpha-1} f(v) d v \\
& =\int_{T}^{\mu} f(v) d v \int_{v}^{\mu} e^{-k t}(t-v)^{\alpha-1} d t \\
& =\int_{T}^{\mu} f(v) d v \int_{0}^{\mu-v} e^{-k(u+v)} u^{\alpha-1} d u \\
& =\int_{T}^{\mu} e^{-k v} f(v) d v \int_{0}^{\mu-v} e^{-k u} u^{\alpha-1} d u \\
& \rightarrow \Gamma(\alpha) k^{-\alpha} \int_{T}^{\infty} e^{-k v} f(v) d v,
\end{aligned}
$$

as $\mu \rightarrow \infty$, the process being justified as in Theorem 1 . This proves the theorem.

Theorem 3. Assume $k>0,0<\alpha<1$. Then

$$
\int_{T}^{\infty} \cos k t_{T} I_{t}^{\alpha} f(t) d t=k^{-\alpha} \int_{T}^{\infty} \cos (k t+\pi \alpha / 2) f(t) d t,
$$

provided that either the right-hand side exists in the Lebesgue sense or $f(t)$ is continuous and the right-hand side convergent, not necessarily absolutely. The same is true when the cosine is replaced by sine throughout.

Proof. We have

$$
\begin{aligned}
\Gamma(\alpha) \int_{T}^{\mu} \cos k t_{T} I_{t}^{\alpha} f(t) d t= & \int_{T}^{\mu} \cos k t d t \int_{T}^{t}(t-v)^{\alpha-1} f(v) d v \\
& =\int_{T}^{\mu} f(v) d v \int_{v}^{\mu}(t-v)^{\alpha-1} \cos k t d t \\
& =k^{-\alpha} \int_{T}^{\mu} f(v) d v \int_{0}^{k(\mu-v)} u^{\alpha-1} \cos (u+k v) d u \\
= & k^{-\alpha}\left\{\int_{T}^{\mu} f(v) \cos k v d v \int_{0}^{k(\mu-v)} u^{\alpha-1} \cos u d u\right. \\
& \left.-\int_{T}^{\mu} f(v) \sin k v d v \int_{0}^{k(\mu-v)} u^{\alpha-1} \sin u d u\right\} \\
\rightarrow & \Gamma(\alpha) k^{-\alpha} \int_{T}^{\infty} \cos (k v+\pi \alpha / 2) f(v) d v,
\end{aligned}
$$


as $\mu \rightarrow \infty$, the process being justified as in Theorem 1 . The corresponding theorem, with cosine replaced by sine, may be proved in a similar way.

It will be noticed that the above proof breaks down when $\alpha=1$. If indeed the theorem were true for $\alpha=1$, we should have as a corollary that

$$
\cos k \mu \int_{T}^{\mu} f(t) d t \rightarrow 0
$$

which is clearly not true in general.

3. Applications of the theorems. We now apply these theorems to the evaluation of the integrals*

$$
\begin{array}{lr}
\int_{0}^{\infty} x^{-n-m} J_{n}(a x) d x, & n>-1 / 2,0<m<1, \\
\int_{0}^{\infty} e^{-k x^{2} x^{n+1} J_{n}(a x) d x,} & n>-1 / 2, k>0, \\
\int_{0}^{\infty} x^{n+1} \cos k x^{2} J_{n}(a x) d x, & -1 / 2<n<1 / 2, k>0, \\
\int_{0}^{\infty} x^{n+1} \sin k x^{2} J_{n}(a x) d x, & -1 / 2<n<1 / 2, k>0 .
\end{array}
$$

It is found that Bessel functions lend themselves readily to the methods of the fractional calculus. W. O. Pennell $\dagger$ has shown that

$$
J_{n}\left(a t^{1 / 2}\right)=(a / 2)^{n-1} \pi^{-1 / 2} t^{-n / 2}{ }_{0} I_{t}^{n-1 / 2} \sin a t^{1 / 2} .
$$

This was proved by expanding $\sin a t^{1 / 2}$ in a power series and integrating fractionally term by term. It holds for all values of $n$, positive and negative, provided we write as usual $I^{-\alpha}=D^{p} I^{p-\alpha}, p$ being an integer such that $p>\alpha \geqq p-1 \geqq 0$, and $D^{p}$ denoting repeated differentiation in the usual sense. If $n>-1 / 2$, we may also derive (9) by taking Poisson's integral

$$
J_{n}(a t)=\frac{2(a t / 2)^{n}}{\Gamma(n+1 / 2) \Gamma(1 / 2)} \int_{0}^{1}\left(1-u^{2}\right)^{n-1 / 2} \cos a t u d u,
$$

and setting $t u=v^{1 / 2}$. This gives

* An evaluation of integrals (6) and (7) is to be found in Watson, Bessel Functions, chap. 13, pp. 393-394; or see Gray and Mathews, Bessel Functions, 1st edition, formulas (163) and (162), or 2d edition, formula (8) on page 66 and formula (19) on page 71. In each of these books references to the original papers of Weber and others are given.

$\dagger$ This Bulletin, vol. 38 (1932), pp. 115-122. 


$$
J_{n}(a t)=\frac{(a t / 2)^{n}}{t^{2 n} \Gamma(n+1 / 2) \Gamma(1 / 2)} \int_{0}^{t^{2}}\left(t^{2}-v\right)^{n-1 / 2} \frac{\cos a v^{1 / 2}}{v^{1 / 2}} d v ;
$$

whence, by changing $t$ into $t^{1 / 2}$, we obtain

$$
J_{n}\left(a t^{1 / 2}\right)=(a / 2)^{n} \pi^{-1 / 2} t^{-n / 2}{ }_{0} I_{t}^{n+1 / 2}\left(t^{-1 / 2} \cos a t^{1 / 2}\right),
$$

a formula equivalent to (9). It is now easy to evaluate the integrals under consideration. In the first place, we have

$$
\begin{aligned}
\int_{0}^{\infty} \frac{J_{n}(a x)}{x^{n+m}} d x & =\frac{1}{2} \int_{0}^{\infty} \frac{J_{n}\left(a t^{1 / 2}\right)}{t^{n / 2+m / 2+1 / 2}} d t \\
& =\frac{1}{2}(a / 2)^{n} \pi^{-1 / 2} \int_{0}^{\infty} \frac{1}{t^{n+m / 2+1 / 2}}{ }_{0} I_{t^{n+1 / 2}} \frac{\cos a t^{1 / 2}}{t^{1 / 2}} d t
\end{aligned}
$$

by (10). Applying Theorem 1, we find that this is equal to

$$
\begin{aligned}
& \frac{1}{2}(a / 2)^{n} \pi^{-1 / 2} \frac{\Gamma(m / 2)}{\Gamma(n+m / 2+1 / 2)} \int_{0}^{\infty} \frac{\cos a t^{1 / 2}}{t^{m / 2+1 / 2}} d t \\
& =(a / 2)^{n} \pi^{-1 / 2} \frac{\Gamma(m / 2)}{\Gamma(n+m / 2+1 / 2)} \int_{0}^{\infty} \frac{\cos a x}{x^{m}} d x \\
& \quad=(a / 2)^{n} \pi^{-1 / 2} \frac{\Gamma(m / 2)}{\Gamma(n+m / 2+1 / 2)} a^{m-1} \Gamma(1-m) \sin \pi m / 2 .
\end{aligned}
$$

Simplifying by routine methods, we obtain finally

$$
\int_{0}^{\infty} \frac{J_{n}(a x)}{x^{n+m}} d x=a^{n+m-1} 2^{-n-m} \frac{\Gamma(1 / 2-m / 2)}{\Gamma(n+m / 2+1 / 2)} .
$$

The same method may be applied to the integral (7). We have

$$
\begin{aligned}
\int_{0}^{\infty} e^{-k x^{2}} x^{n+1} J_{n}(a x) d x & =\frac{1}{2} \int_{0}^{\infty} e^{-k t} t^{n / 2} J_{n}\left(a t^{1 / 2}\right) d t \\
& =\frac{1}{2}(a / 2)^{n} \pi^{-1 / 2} \int_{0}^{\infty} e^{-k t} I_{0} I^{n+1 / 2}\left(t^{-1 / 2} \cos a t^{1 / 2}\right) d t,
\end{aligned}
$$

by (10). Applying Theorem 2 we find that this is equal to

$$
\begin{aligned}
& \frac{1}{2}(a / 2)^{n} \pi^{-1 / 2} k^{-(n+1 / 2)} \int_{0}^{\infty} e^{-k t} t^{-1 / 2} \cos a t^{1 / 2} d t \\
& =(a / 2)^{n} \pi^{-1 / 2} k^{-(n+1 / 2)} \int_{0}^{\infty} e^{-k x^{2}} \cos a x d x
\end{aligned}
$$




$$
=(a / 2)^{n} \pi^{-1 / 2} k^{-(n+1 / 2)} e^{-a^{2} / 4 k} \int_{0}^{\infty} e^{-k x^{2}} d x,
$$

as is readily proved by a contour integration method.* Hence, finally,

$$
\int_{0}^{\infty} e^{-k x^{2}} x^{n+1} J_{n}(a x) d x=(2 k)^{-n-1} a^{n} e^{-a^{2} / 4 k}
$$

Similarly, by using Theorem 3, we find that

$$
\begin{aligned}
& \int_{0}^{\infty} x^{n+1} \cos k x^{2} J_{n}(a x) d x \\
& \quad=2^{1 / 2}(2 k)^{-n-1} a^{n}\left\{\cos \left(\pi n / 2+\pi / 4-a^{2} / 4 k\right)-\sin \left(\pi n / 2+\pi / 4-a^{2} / 4 k\right)\right\}
\end{aligned}
$$

and that

$$
\begin{aligned}
& \int_{0}^{\infty} x^{n+1} \sin k x^{2} J_{n}(a x) d x \\
& \quad=2^{1 / 2}(2 k)^{-n-1} a^{n}\left\{\cos \left(\pi n / 2+\pi / 4-a^{2} / 4 k\right)+\sin \left(\pi n / 2+\pi / 4-a^{2} / 4 k\right)\right\},
\end{aligned}
$$

provided $-1 / 2<n<1 / 2$. These examples will serve to indicate some of the uses of the theorems given above.

Acadia University

*Whittaker and Watson, Modern Analysis, 4th edition, p. 114, example 3. 